\title{
Respiratory pathogens in patients with acute exacerbation of non-cystic fibrosis bronchiectasis from a developing country
}

\author{
Shayan Shahid, ${ }^{1}$ Kauser Jabeen, ${ }^{2}$ Nousheen Iqbal, ${ }^{3,4}$ Joveria Farooqi, ${ }^{2}$ Muhammad Irfan ${ }^{3}$ \\ ${ }^{1}$ Medical College, Aga Khan University, Hospital, Karachi; ${ }^{2}$ Department of Pathology and Laboratory Medicine, Aga \\ Khan University, Karachi; ${ }^{3}$ Section of Pulmonary and Critical Care Medicine, Department of Medicine, Aga Khan \\ University, Karachi; ${ }^{4}$ Jinnah Medical and Dental College, Karachi, Pakistan
}

\begin{abstract}
Bronchiectasis unrelated to cystic fibrosis (non-CF bronchiectasis) has become a major respiratory disease in developing nations. The dilated mucus filled airways promote bacterial overgrowth followed by chronic infection, bronchial inflammation, lung injury and re-infection. Accurate pathogen identification and antimicrobial susceptibility allowing appropriate treatment, in turn, may break this vicious cycle. This study aimed to gain kowledge
\end{abstract}

Correspondence: Prof. Muhammad Irfan, Section of Pulmonary and Critical Care Medicine, Department of Medicine, Aga Khan University, Stadium Road, Karachi, Pakistan.

Tel. + 92.21.34864664.

E-mail: muhammad.irfan@aku.edu

Key words: Bronchiectasis; etiology; infection; drug resistance.

Ethics approval and consent to participate: The study was approved by the ethical review committee (ERC) of Aga Khan University (AKU). (ERC \# (5097-MED-ERC-17, dated 27th November 2017).

Consent for publication: Not applicable

Availability of data and materials: The datasets used and/or analyzed during the current study are available from the corresponding author on reasonable request.

Conflict of interest: The authors declare that they have no competing interests, and all authors confirm accuracy.

Authors' contributions: MI, study supervisor. All the authors made a substantive intellectual contribution, have read and approved the final version of the manuscript and agreed to be accountable for all aspects of the work.

Received for publication: 18 January 2021

Accepted for publication: 14 April 2021.

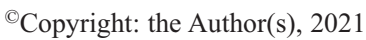

Licensee PAGEPress, Italy

Monaldi Archives for Chest Disease 2021; $91: 1771$

doi: 10.4081/monaldi.2021.1771

This article is distributed under the terms of the Creative Commons Attribution Noncommercial License (by-nc 4.0) which permits any noncommercial use, distribution, and reproduction in any medium, provided the original author(s) and source are credited. about the spectrum and antimicrobial spectrum of pathogen yielded from respiratory specimens in adult patients with acute exacerbation of non-cystic fibrosis (CF) bronchiectasis. This cross-sectional study was performed at the pulmonology clinics of the Aga Khan University, Karachi, Pakistan from 2016-2019. Respiratory specimens were collected from adult patients with acute exacerbation of non-CF bronchiectasis presenting in pulmonology clinics. Microbial cultures were performed using standard methodology. Susceptibility testing was performed and interpreted using Clinical Laboratory Standard Institute criteria. A total of 345 positive cultures from 160 patients presenting with acute exacerbation were evaluated. The most frequent organisms were Pseudomonas aeruginosa $(\mathrm{n}=209)$ followed by Hemophilus influenzae $(\mathrm{n}=40)$ and Staphylococcus aureus $(\mathrm{n}=24)$. High rates of antimicrobial resistance were found in all these pathogens. Proportion of Pseudomonas aeruginosa strains resistant to ciprofloxacin, imipenem, ceftazidime and piperacillin-tazobactam were $27.1 \%$, $16.8 \%, 14.8 \%$ and $13.1 \%$ respectively. $65 \%$ of Hemophilus influenzae strains were resistant to cotrimoxazole and ciprofloxacin and $66.7 \%$ of Staphylococcus aureus strains were resistant to methicillin. High antimicrobial resistance in non-CF bronchiectasis patients against commonly used antimicrobials is a concern and highlight need for urgent community level interventions to improve clinical outcome in these patients.

\section{Introduction}

Bronchiectasis is a suppurative lung disease which is characterized by inflamed and dilated bronchi [1]. Bronchiectasis unrelated to cystic fibrosis (non-CF bronchiectasis) has become a major primary respiratory disease in developing nations [2). It is a complex disease which gives rise to repeated chest infections due to a vicious cycle. The dilated airways filled with mucus promote growth of variety of organisms which is followed by chronic infection, bronchial inflammation, lung injury and re-infection [3]. It is a cause of significant morbidity and mortality in adults and has multiple etiologies (e.g. autoimmune disease, cilia abnormalities, obstruction, infectious diseases, allergic bronchopulmonary aspergillosis (ABPA), connective tissue disease) and phenotypic features [4,5]. The mortality rate ranged from $10 \%$ to $16 \%$ over a 4 -year observation period in a study conducted in USA, with culture positive for Pseudomonas aeruginosa in sputum, low BMI, male sex, advanced age and COPD identified as risk factors for mortality [4]. Therefore, identifying the causative organism and its sensitivity may allow appropriate treatment, in turn, breaking the vicious cycle [3].

Gram negative bacteria are the most frequently seen organ- 
isms in the sputum of bronchiectasis patients [4]. Studies conducted in adult populations of Spain, Greece, Thailand, Australia, UK and China identified Haemophilus influenzae and Pseudomonas aeruginosa as the most commonly isolated bacteria followed by Streptococcus pneumoniae, Moraxella catarrhalis and Staphylococcus aureus [1,5-11].

India conducted a first of its kind bronchiectasis study in a lower-middle income country in 2019 that showed $P$. aeruginosa to be the most common isolated pathogen followed by Enterobacteriaceae, S. aureus, $M$. catarrhalis and $H$. influenzae highlighting significant variation from Europe [12]. A cohort study conducted in a tertiary care hospital in Karachi, Pakistan also reported $P$. aeruginosa as the most commonly cultured organism (36.2\%) followed by $M$. catarrhalis and $H$. influenzae [13].

Exacerbations in non-CF bronchiectasis are a significant cause of morbidity [14]. It has been hypothesized that the onset might be triggered by a change in airway bacterial composition, or spread of same species to new regions of the lung [15]. Factors that contribute to pathogenesis of bronchiectasis can also cause exacerbations which include neurological disorders, poor nutrition, poverty etc. but it is assumed that a pathogen is always involved at some point [2]. P. aeruginosa has been seen to be the most common pathogen isolated in exacerbations $[16,17]$ followed by $H$. influenzae [18], S. pneumoniae, S. aureus, M. catarrhalis, Escherichia coli and Serratia species in that order [14,15]. A double-blind randomized control trial in New Zealand in 2012 also noted a similar trend with $H$. influenzae being the most common isolated pathogen [19]. Sputum cultures from patients with bronchiectasis exacerbation, and on treatment in the ICU for acute respiratory failure, yielded $P$. aeruginosa most frequently followed by $S$. pneumoniae and $H$. influenzae [20].

In other studies conducted in Spain assessing the bacterial spectrum and antimicrobial resistance patterns in exacerbations, $P$. aeruginosa was noted to be the most common bacterial pathogen isolated, followed by S. pneumoniae, H. influenzae, S. aureus and Enterobacteriaceae $[21,22]$. P. aeruginosa was also seen to be the most antibiotic resistant organism with isolates being ciprofloxacin resistant and multi-drug resistant (not susceptible to at least 1 agent in 3 or more antimicrobial classes) $[21,22]$. Many of the $S$. aureus isolates were also found to be methicillin resistant $[21,22]$.

Little has been published specifically on the spectrum and antibiotic resistance pattern of bacteria isolated from sputum cultures of adult patients with exacerbations of non-CF bronchiectasis from developing countries. Due to the rampant use of antibiotics, antimicrobial resistance is becoming a prominent concern for clinicians around the world, especially in developing countries. One of the main culprits for this situation is the ease with which antibiotics can be purchased over the counter, and an increased propensity of the clinicians for prescribing antibiotics. Data on spectrum of etiology, organisms and resistance in non-CF bronchiectasis is almost non-existent in the subcontinent and this study intends to bridge this gap. This study was conducted to identify the microbial agents present in sputum specimen and their resistance pattern in non-CF bronchiectasis patients with acute exacerbations.

\section{Materials and Methods}

This was a cross sectional study from the Aga Khan University Hospital (AKUH), Karachi, Pakistan (tertiary care hospital) over a 4-year period between 2016 and 2019. Adult patients ( $>18$ years) diagnosed with non-CF bronchiectasis on high resolution $\mathrm{CT}$
(HRCT) scan, who presented to outpatient pulmonology clinic with acute exacerbation, were included. Patients with a diagnosis of cystic fibrosis (CF) (or suspected CF) as well as patients who did not have HRCT scan were excluded. An exacerbation of bronchiectasis was defined as a clinical diagnosis by the physician when the patient presented with one or more of the following symptoms: uncontrolled cough, increased sputum volume, worsened sputum purulence, aggravated dyspnea, fatigue, fever, and hemoptysis [23].

Clinical findings, etiology, microbiological culture of respiratory specimen samples and drug susceptibility was recorded on predesigned performa. All microbial cultures were conducted in the AKUH laboratory that is accredited with the College of American Physicians (CAP). All specimens were processed and reported using CAP standards. Susceptibility testing was performed and interpreted using Clinical Laboratory Standard Institute criteria. Sensitivity testing was carried out using the agar disc diffusion method. Vancomycin susceptibility testing was reported based on minimum inhibitory concentrations.

The study was approved by Ethical review committee (ERC) of Aga Khan University Hospital (ERC \# (5097-MED-ERC-17, dated $27^{\text {th }}$ November 2017).

The data was entered and analyzed using SPSS version 22.0. Baseline characteristics, etiology, frequency of isolation in cultures and resistance pattern of organisms were assessed and presented in percentages.

\section{Results}

A total of 160 patients who presented to AKUH pulmonology clinics with acute exacerbations of non-CF bronchiectasis and fulfilled the inclusion criteria were studied. Table 1 shows the demographic data of the studied population. Women $(56.9 \%)$ and the age group $>60(46.9 \%)$ predominated. Figure 1 summarizes the etiology of non-CF bronchiectasis in study population. The most common etiology seen was Post TB $(n=65)$ which comprised of $40.6 \%$ of the sample followed by idiopathic $(n=50)$ and ABPA $(n=18)$. A total of 345 positive respiratory specimen culture results from 160 patients, in the specified time duration, were evaluated. This equates to a mean of 2.2 sputum cultures per patient. A total of 20 different organisms were isolated from these patients (Table 2). The most frequently isolated organisms were P. aeruginosa

Table 1. Demographics of bronchiectasis patients.

\begin{tabular}{lcc} 
Characteristics & Values & Range \\
Age (years) & $\mathrm{n}(\%)$ & \\
$18-30$ & $25(15.6 \%)$ & \\
$31-45$ & $32(20 \%)$ & \\
$46-60$ & $28(17.5 \%)$ & \\
$>60$ & $75(46.9 \%)$ & \\
Age (mean \pm SD) & $54 \pm 19$ & $18-101$ \\
Sex & $91(56.9 \%)$ & \\
Female & $69(43.1 \%)$ & \\
Male & $1.3: 1$ & \\
Female:Male ratio & $22.03 \pm 6.63$ & $14.6-42.3$ \\
\hline BMI (mean \pm SD) & $40(25 \%)$ & \\
History of influenza vaccination & $2.13 \pm 1.9$ & $1-5$ \\
\hline Rate of exacerbation /year (mean \pm SD) & & \\
\hline
\end{tabular}


from 209 samples $(60.6 \%), H$. influenzae from 40 patients (11.6\%), S. aureus from 24 patients (7.0\%), Klebsiella pneumoniae from 20 patients $(5.8 \%)$, and S. pneumoniae from 19 patients (5.5\%). Relatively rare organisms like Aeromonas species, Burkholderia cepacia, Chryseobacterium species, Nocardia species and Stenotrophomonas maltophilia were also seen. Twenty-seven $(7.8 \%)$ cultures had a polymicrobial growth. Figure 2 shows resistance patterns (including both resistant and intermediately resistant strains) of the four most frequently isolated organisms. P. aeruginosa isolates demonstrated highest resistance to aztreonam $(32.0 \%)$ followed by ciprofloxacin $(27.1 \%)$ and gentamicin $(25.6 \%)$, while $44(21 \%)$ of P. aeruginosa isolates were multi-drug resistant (not susceptible to at least 1 agent in 3 or more antimicrobial classes). $H$. influenzae isolates showed highest resistance to cotrimoxazole (65.0\%) and ciprofloxacin (64.1\%). S. aureus isolates were all resistant to penicillin (100\%), followed by levofloxacin $(83.3 \%)$, erythromycin $(75 \%)$, gentamicin $(50 \%)$, cotrimoxazole $(29.2 \%)$, tetracycline $(21.7 \%)$, clindamycin (20.8\%) while $66.7 \%$ were categorized as MRSA. Vancomycin resistance was not observed. All of $K$. pneumoniae isolates were resistant to ampicillin, and $81.3 \%$ of isolates were resistant to cefuroxime followed by ciprofloxacin and cotrimoxazole. S. pneumoniae isolates were mostly sensitive to beta lactam but showed highest resistance to cotrimoxazole $(72.2 \%)$ followed by tetracycline $(52.9 \%)$ and erythromycin $(50 \%)$.

\section{Discussion}

Our study demonstrated that the majority of the organisms isolated in the respiratory specimens in our patients' population were gram negative $(86 \%)$ concordant with the studies conducted in other parts of the world [4]. P. aeruginosa was by far the most frequently isolated organism $(60.6 \%)$ followed by $H$. influenzae
(11.6\%). Gram-positive organisms were less common with $S$. aureus and S. pneumoniae present in $7.0 \%$ and $5.5 \%$ of the samples respectively. These results are consistent with previous studies conducted on patients with bronchiectasis exacerbation [16,17,19,21-23]. Additionally, a wide range of bacteria were iso-

Table 2. Bacterial species isolated from patients. The culture results were organized by organism cultured.

\begin{tabular}{lcc} 
Organism & Frequency $(\mathbf{n = 3 4 5})$ & $\%$ \\
Pseudomonas aeruginosa & 209 & 60.6 \\
Hemophilus influenzae & 40 & 11.6 \\
\hline Staphylococcus aureus & 24 & 7.0 \\
Klebsiella pneumoniae & 20 & 5.8 \\
\hline Streptococcus pneumoniae & 19 & 5.5 \\
Acinetobacter species & 12 & 3.5 \\
\hline Hemophilus parainfluenzae & 12 & 3.5 \\
Escherichia coli & 11 & 3.2 \\
\hline Moraxella catarrhalis & 6 & 1.7 \\
Serratia species & 3 & 0.9 \\
\hline Staphylococcus species (not aureus) & 3 & 0.9 \\
Enterococcus species & 2 & 0.6 \\
\hline Nocardia asteroides & 2 & 0.6 \\
Pseudomonas species (not aeruginosa) & 2 & 0.6 \\
\hline Stenotrophomonas maltophilia & 2 & 0.6 \\
Aeromonas species & 1 & 0.3 \\
\hline Burkholderia cepacia & 1 & 0.3 \\
Chryseobacterium species & 1 & 0.3 \\
\hline Enterobacter species & 1 & 0.3 \\
\hline
\end{tabular}

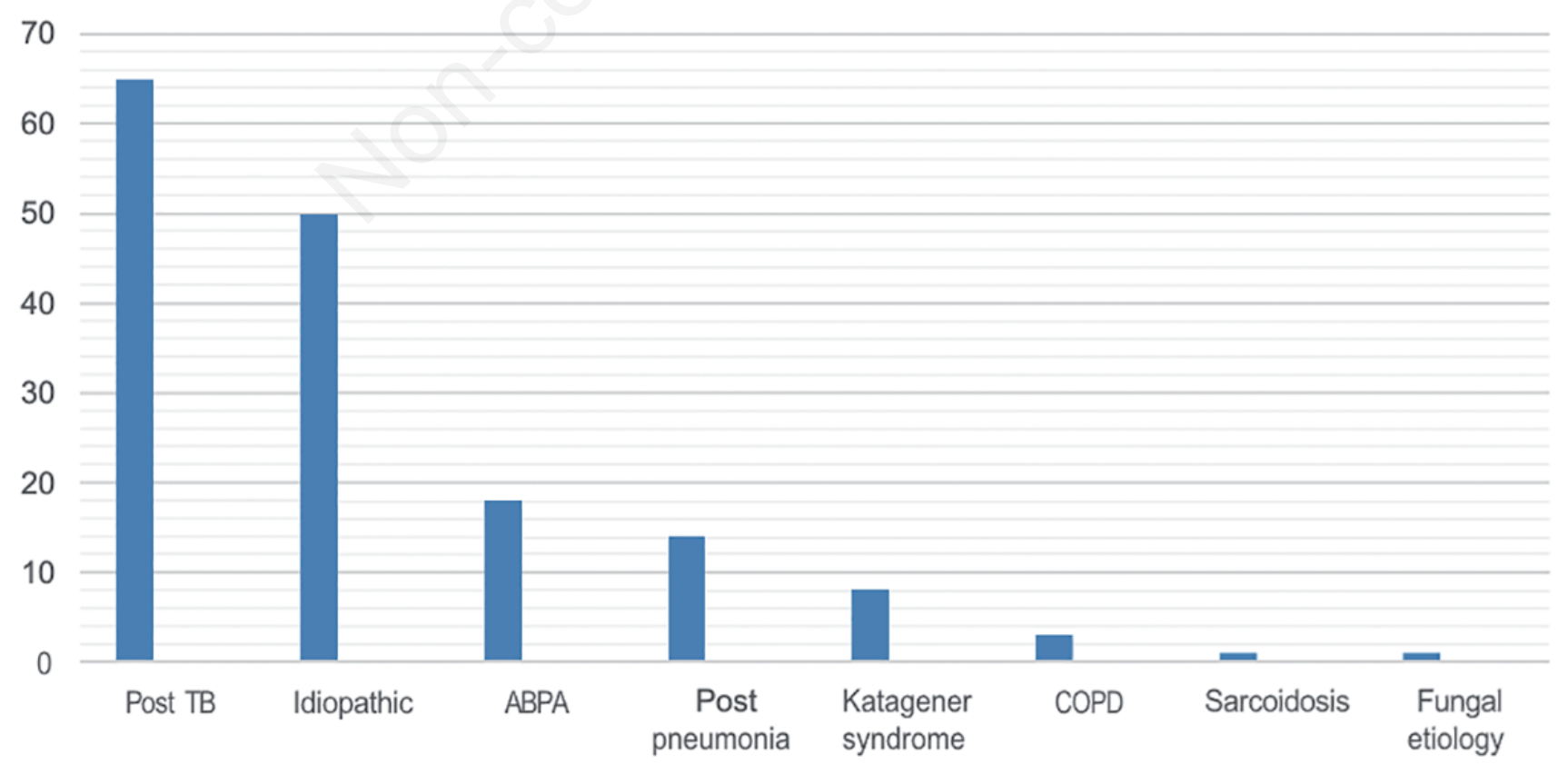

Figure 1. Etiology as defined at recruitment. 


\section{Hemophilus influenzae} $(n=209)$

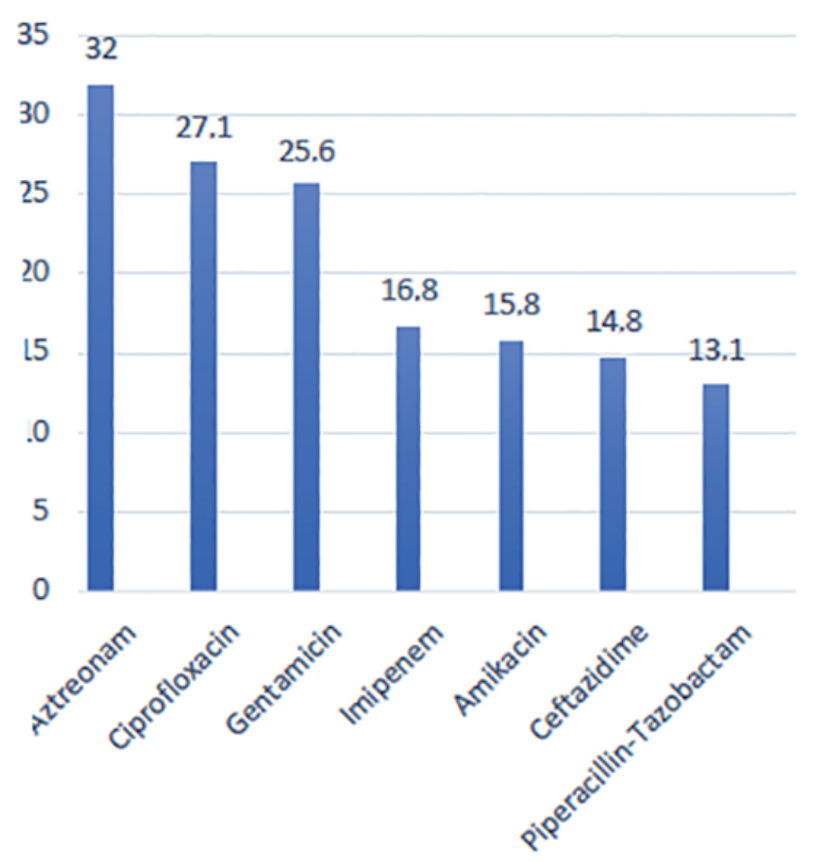

\section{Streptococcus pneumoniae} ( $n=19)$

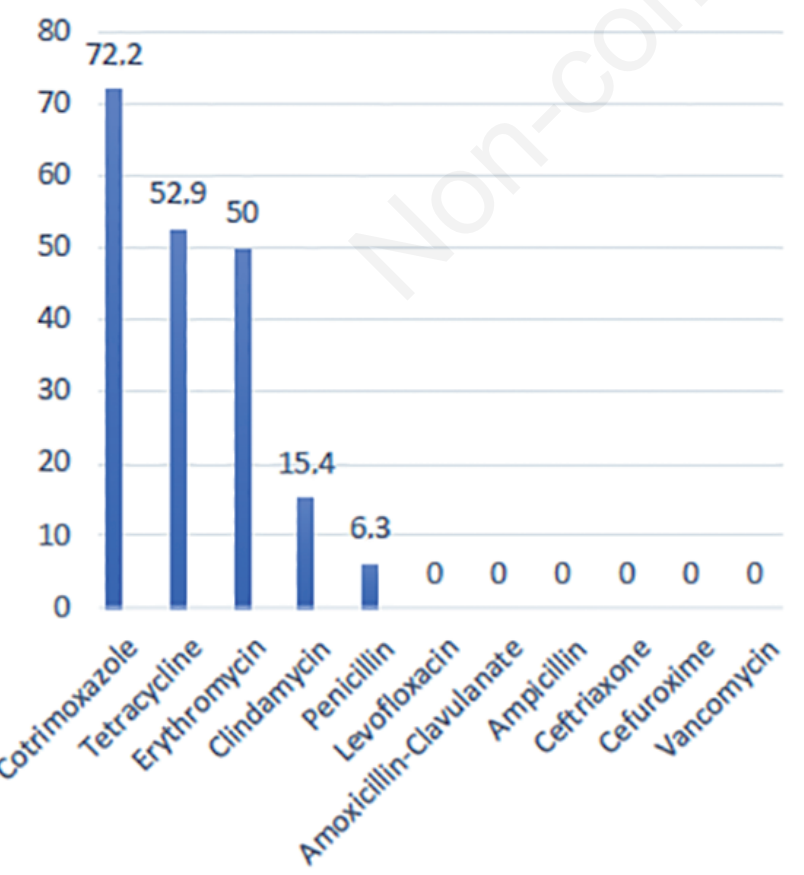

$(n=40)$

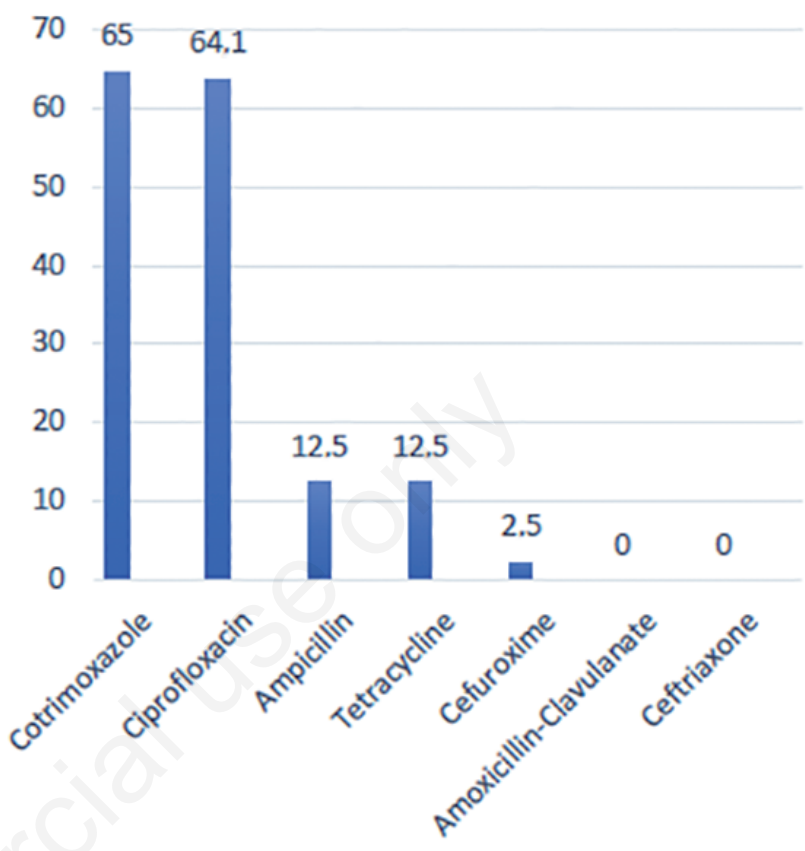

Klebsiella pneumoniae $(n=20)$

120
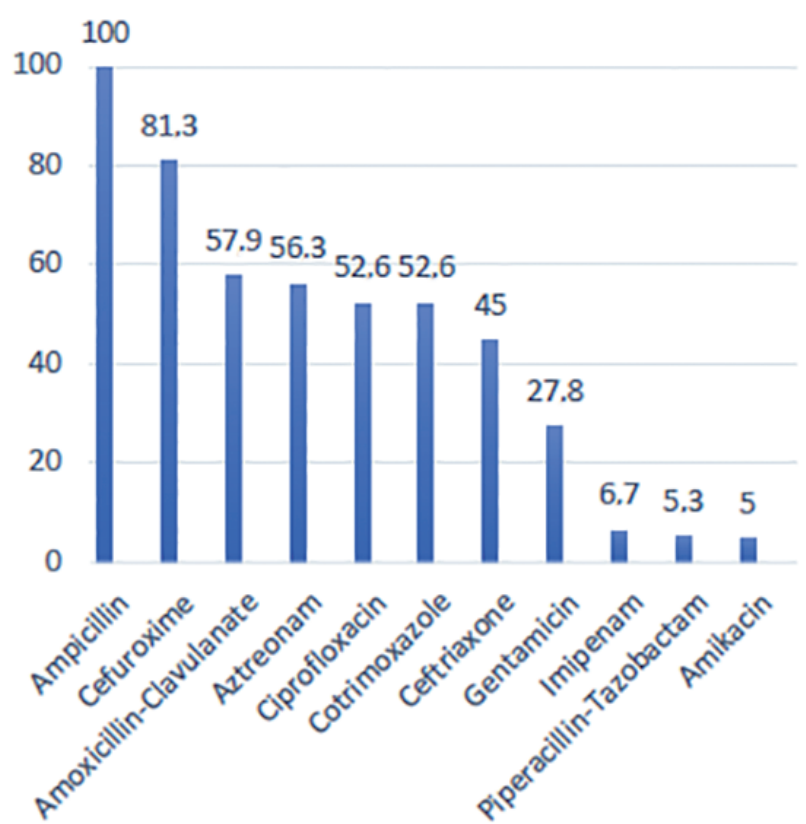

Figure 2. Antimicrobial resistance (\% resistant isolates) in bacteria isolated from respiratory samples from non-CF bronchiectasis patients. 
lated that included rare organisms like Aeromonas species, Stenotrophomonas maltophilia and Chryseobacterium species.

Clinical features, effect on lung function and disease severity vary with different bacteria. P. aeruginosa infections have been connected to worse pulmonary function, a greater spread of disease (extent of lung involvement), and poor quality of life [24,25]. Patients, whose cultures grow $P$. aeruginosa in acute exacerbation of bronchiectasis, are noted to have significantly higher number of hospital admissions in a year [20]. This is coupled with a greater degree of bronchial wall thickening and dilatation, as well as greater degree of small airway disease [26]. P. aeruginosa colonization has also been seen to be an independent predictor of mortality in bronchiectasis [27]. This is alarming as a major portion of patients with exacerbation in our study cultured $P$. aeruginosa. This calls for infection control measures to be taken for prevention, as well as improved empirical and definitive treatment regimens [27]. In addition, $H$. influenzae has been linked to significantly worsened CT scan findings [26].

$P$. aeruginosa strains had high resistance to aztreonam, ciprofloxacin and gentamicin. Ciprofloxacin resistance $(27.1 \%)$ in our study was higher than has been observed in a previous study (18.4\%) [21]. 44 (21\%) of the P. aeruginosa isolates (12.8\% of all specimens) were multi- drug resistant (not susceptible to at least 1 agent in 3 or more antimicrobial classes). This is slightly higher than MDR $P$. aeruginosa isolates seen in previous studies in which MDR $P$. aeruginosa comprised of $7.9 \%$ and $6.6 \%$ of samples $[21,22]$. Around two-thirds of the $S$. aureus isolates in our study were methicillin resistant which is twice as high as $25 \%$ and $35.3 \%$ seen in other studies [21,22]. This difference in antibiotic resistance can be attributed to unrestricted supply of antibiotics in Pakistan (a developing/LMI country), excessive prescription due to patient demand, self-medication (28) for conditions that do not necessarily require antibiotics (flu, cough, abdominal pain, skin infections, fever, etc.) [29], lack of basic knowledge about antibiotics (that includes correct dosage, indications etc.), illiteracy and failure to complete the antibiotic course after symptoms subside [30,31].

$H$. influenzae isolates showed high resistance to cotrimoxazole $(65 \%)$ and ciprofloxacin $(64.1 \%)$. K. pneumoniae isolates had high resistance to cefuroxime $(81.3 \%)$ followed by ciprofloxacin and cotrimoxazole. S. pneumoniae isolates showed highest resistance to cotrimoxazole (72.2\%) followed by tetracycline and erythromycin. Unfortunately, there is lack of data available to compare for resistance patterns of these organisms in bronchiectasis exacerbations throughout the world. In fact, to our knowledge, this is the first study to describe resistance pattern of all isolated organisms in bronchiectasis exacerbation. Antimicrobial resistance of these organisms has been studied in Pakistan for other respiratory infections including pneumonia, acute exacerbation of chronic bronchitis etc. H. influenzae isolates have been seen to exhibit lower rate of resistance to antimicrobials in the past with chloramphenicol and cotrimoxazole being notable exceptions [32,33]. K. pneumoniae has shown high resistance rates to ampicillin, cotrimoxazole and amoxicillin/clavulanic acid [34]. High to moderate resistance to cotrimoxazole and erythromycin has been seen in S. pneumoniae isolates [32-35]. S. aureus has been reported to be highly resistant to erythromycin and levofloxacin [34]. These reported antimicrobial resistance patterns are consistent with the findings of our study.

Our study covers data from one tertiary care hospital in Pakistan. Since it is a private hospital, the patient population belongs to upper-middle class to upper class members of the society. Thus, it is not a true representation of the entire population of the city. Moreover, due to its reputation in the country, the pulmonology clinics receive relatively complicated cases and there- fore the severity of disease studied may be different from that seen across the country. The study also covers a relatively small sample size of 160 patients.

\section{Conclusions}

$P$. aeruginosa was the most frequently isolated organism in the respiratory specimens of patients diagnosed with an acute exacerbation of non-cystic fibrosis bronchiectasis; $21 \%$ of the P. aeruginosa strains were multi-drug resistant. High resistance of $P$. aeruginosa strains to anti-pseudomonal antimicrobials was noted. This is a source of concern as it is the most frequently isolated organism and has been associated with poor prognosis in patients.

Since this study involves only one tertiary care hospital which caters to only one subset of the population, a multicenter country wide study with a larger sample size is needed to further evaluate the bacterial etiology and resistance pattern in the population of Pakistan.

\section{References}

1. Dimakou K, Triantafillidou C, Toumbis M, et al. Non CFbronchiectasis: Aetiologic approach, clinical, radiological, microbiological and functional profile in 277 patients. Respir Med 2016;116:1-7.

2. Chang A, Bilton D. Exacerbations in cystic fibrosis: Non-cystic fibrosis bronchiectasis. Thorax 2008;63:269-76.

3. Foweraker J, Wat D. Microbiology of non-CF bronchiectasis. Eur Respir Mon 2011;52:68-96.

4. McShane PJ, Naureckas ET, Tino G, Strek ME. Non-cystic fibrosis bronchiectasis. Am J Respir Crit Care Med 2013;188:647-56.

5. Pasteur MC, Helliwell SM, Houghton SJ, et al. An investigation into causative factors in patients with bronchiectasis. Am J Respir Crit Care Med 2000;162:1277-84.

6. Angrill J, Agusti C, De Celis R, et al. Bacterial colonisation in patients with bronchiectasis: microbiological pattern and risk factors. Thorax 2002;57:15-9.

7. King PT, Holdsworth SR, Freezer NJ, et al. Microbiologic follow-up study in adult bronchiectasis. Respir Med 2007; 101:1633-8.

8. Macfarlane J, McAlinden P, De Soyza A. Longitudinal study of sputum microbiology in adult non-CF bronchiectasis. Thorax 2010;65:A177-8.

9. Palwatwichai A, Chaoprasong C, Vattanathum A, et al.Clinical, laboratory findings and microbiologic characterization of bronchiectasis in Thai patients. Respirology 2002;7:63-6.

10. Olveira C, Padilla A, Martínez-García M-Á, et al. Etiology of bronchiectasis in a cohort of 2047 patients. An analysis of the Spanish historical bronchiectasis registry. Arch Bronconeumol 2017;53:366-74.

11. Chandrasekaran R, Mac Aogáin M, Chalmers JD, et al. Geographic variation in the aetiology, epidemiology and microbiology of bronchiectasis. BMC Pulm Med 2018;18:83.

12. Dhar R, Singh S, Talwar D, Mohan M, et al. Bronchiectasis in India: results from the European Multicentre Bronchiectasis Audit and Research Collaboration (EMBARC) and Respiratory Research Network of India Registry. Lancet Global Health 2019;7:e1269-e79. 
13. Sharif N, Baig MS, Sharif S, Irfan M. Etiology, clinical, radiological, and microbiological profile of patients with non-cystic fibrosis bronchiectasis at a tertiary care hospital of Pakistan. Cureus 2020;12:e7208.

14. Murray MP, Turnbull K, MacQuarrie S, Hill AT. Assessing response to treatment of exacerbations of bronchiectasis in adults. Eur Respira J 2009;33:312-8.

15. Tunney MM, Einarsson GG, Wei L, et al. Lung microbiota and bacterial abundance in patients with bronchiectasis when clinically stable and during exacerbation. Am J Respir Crit Care Med 2013;187:1118-26.

16. Finklea JD, Khan G, Thomas S, et al. Predictors of mortality in hospitalized patients with acute exacerbation of bronchiectasis. Respir Med 2010;104:816-21.

17. Gao Y, Guan W, Zhu Y, et al. [Respiratory pathogen spectrum in pulmonary exacerbation of bronchiectasis in adults and its association with disease severity].[Article in Chinese]. Zhonghua Jie He He Hu Xi Za Zhi 2019;42:254-61.

18. Guan W-J, Gao Y-H, Xu G, et al. Inflammatory responses, spirometry, and quality of life in subjects with bronchiectasis exacerbations. Respir Care 2015;601180-9.

19. Wong C, Jayaram L, Karalus N, et al. Azithromycin for prevention of exacerbations in non- cystic fibrosis bronchiectasis (EMBRACE): a randomised, double-blind, placebo-controlled trial. Lancet.2012;380:660-7.

20. Çiftci F, Mülazimoglu DD, Erol S, et al. Effect of sputum bacteriology on the prognosis of patients with acute exacerbations of bronchiectasis in the intensive care unit. Eurasian $\mathrm{J}$ Pulmonol 2018;20:85.

21. Polverino E, Rosales-Mayor E, Benegas M, et al. Pneumonic and non-pneumonic exacerbations in bronchiectasis: clinical and microbiological differences. J Infect 2018;77:99- 106.

22. Menéndez R, Méndez R, Polverino E, et al. Risk factors for multidrug-resistant pathogens in bronchiectasis exacerbations. BMC Infect Dis 2017;17:659.

23. Lonni S, Chalmers JD, Goeminne PC, et al. Etiology of noncystic fibrosis bronchiectasis in adults and its correlation to disease severity. Ann Am Thorac Soc 2015;12:1764-70.

24. Wilson C, Jones P, O'leary C, et al. Effect of sputum bacteriol- ogy on the quality of life of patients with bronchiectasis. Eur Respir J 1997; 10:1754-60.

25. Davies G, Wells A, Doffman S, et al. The effect of Pseudomonas aeruginosa on pulmonary function in patients with bronchiectasis. Eur Respir J 2006;28:974-9.

26. Miszkiel KA, Wells AU, Rubens MB, et al. Effects of airway infection by Pseudomonas aeruginosa: a computed tomographic study. Thorax 1997;52:260-4.

27. Loebinger M, Wells A, Hansell D, et al. Mortality in bronchiectasis: a long-term study assessing the factors influencing survival. Eur Respir J 2009;34:843-9.

28. Naing D, Anderios F, Lin Z. Geographic and ethnic distribution of P. knowlesi infection in Sabah, Malaysia. Int J Collab Res Inter Med Public Health 2011;3:391-400.

29. Aslam M, Mirza T, Muhammad A, Sarwat J. Self-medication with antibiotics among secondary and tertiary level students in Karachi, Pakistan: A cross sectional study. Inventi Rapid: Pharmacy Practice 2013;2013:1-5.

30. Atif M, Asghar S, Mushtaq I, et al. What drives inappropriate use of antibiotics? A mixed methods study from Bahawalpur, Pakistan. Infect Drug Resist 2019;12:687.

31. Ali I, Khan AU. Self-medication of antibiotics: A perspective on alarming situation in Peshawar, Khyber Pakhtunkhwa, Pakistan. Arch Pharmacy Pract 2015;6:68.

32. Zafar A, Hasan R, Nizamuddin S, et al. Antibiotic susceptibility in Streptococcus pneumoniae, Haemophilus influenzae and Streptococcus pyogenes in Pakistan: a review of results from the Survey of Antibiotic Resistance (SOAR) 2002-15. J Antimicrob Chemother 2016;71:i103-9.

33. Zafar A, Hussain Z, Lomama E, et al. Antibiotic susceptibility of pathogens isolated from patients with community-acquired respiratory tract infections in Pakistan--the active study. J Ayub Med Coll 2008;20:7.

34. Ali I, Butt M. Antibiotic susceptibility pattern of bacterial isolates from patients of respiratory tract infection at 43 centers in Punjab, Pakistan. Clin Exp Pharmacol 2017;7:229.

35. Shah SN, Ullah B, Basit A, et al. Prevalence and susceptibility patterns of bacteria causing respiratory tract infections in North Waziristan, Pakistan. Pak J Pharm Sci 2016;29:S701-6. 